\title{
Progress and Development Trend of Space Intelligent Robot Technology
}

\author{
Zhihong Jiang $\mathbb{D}^{1,2,3}$ Xiaolei Cao $\mathbb{D}^{1,2,3}$ Xiao Huang $\mathbb{D}^{1,2,3}$ Hui Li $\mathbb{D}^{1,2,3}$ \\ and Marco Ceccarelli ${ }^{4}$ \\ ${ }^{1}$ School of Mechatronic Engineering, Beijing Institute of Technology, Beijing 100081, China \\ ${ }^{2}$ Advanced Innovation Center for Intelligent Robots and Systems, Beijing Institute of Technology, Beijing 100081, China \\ ${ }^{3}$ Key Laboratory of Biomimetic Robots and Systems of Chinese Ministry, Beijing Institute of Technology, Beijing 100081, China \\ ${ }^{4}$ Department of Industrial Engineering, University of Rome Tor Vergata, Via del Politecnico 1, 00133 Roma, Italy
}

Correspondence should be addressed to Xiao Huang; 7520200120@bit.edu.cn and Hui Li; lihui2011@bit.edu.cn

Received 24 August 2021; Accepted 4 January 2022; Published 25 January 2022

Copyright (c) 2022 Zhihong Jiang et al. Exclusive Licensee Beijing Institute of Technology Press. Distributed under a Creative Commons Attribution License (CC BY 4.0).

\begin{abstract}
Since space intelligent robots are not restricted by physiological conditions, it is an attractive choice for the development of automation technology to use them for space exploration and utilization. It is currently the key development direction of the major space powers over the world. This paper first investigates the robotic manipulators and humanoid robot systems for space station applications and reviews theories and methods for robots to achieve large-range stable motion and intelligent dexterous manipulation. Then, the intelligent robot systems for on-orbit satellite maintenance are reviewed, and the related technologies of multirobot collaboration are analyzed. Finally, we investigate the intelligent robot systems for on-orbit assembly of large-scale spatial structures and summarize the technologies of modular assembly and on-orbit manufacture. Overall, this paper reviews the technological progress and development trends of space robots, which provides a good reference for further technical research in this field.
\end{abstract}

\section{Introduction}

It is an inevitable choice for the development of space automation technology to use space intelligent robots to realize space exploration and space resource utilization [1]. Besides the International Space Station (ISS), China started with the successful launch of the Tianhe Core Module in 2021 and intends to build a large-scale, long-term manned national space laboratory during 2021-2022 and gradually develops China's Space Station (CSS). At present, most of the operations in the ISS rely on human astronauts. However, in the mid-to-longterm microgravity environment, astronauts may face various physiological and pathological problems, which will seriously endanger the health of them [2]. Meanwhile, there are huge risks of health and life safety for them when performing tasks outside the cabin.

In addition, since the Soviet Union successfully launched the first satellite of mankind in 1957, mankind has launched nearly 7000 satellites into space, of which about $10 \%$ have failed to orbit correctly or failed in the initial stage. In the harsh space environment, even with the high-reliability design, many satellites still fail, and most of them are high-value high-orbit satellites such that they are difficult to be maintained. In the future, with the in-depth development of space resource utilization, large-aperture antennas, large-diameter optical devices, largescale solar power stations, and other large space structures are important development goals of space utilization and development in the world, while on-orbit assembly and maintenance of these mechanisms will mainly rely on space intelligent robots. Therefore, it is of great social significance and economic benefits to develop and use space robots to realize on-orbit construction, assembly, and maintenance of space stations, satellites, and large space structures.

However, the harsh space environment, including microgravity, complex illumination, and strong radiation in space, poses challenges for space robots in large-range stable motion, high-precision dexterous and safe manipulation, precision sensing, and high-precision measurement. Therefore, this paper will 
review, analyze, and summarize the technical progress and future development trend of space intelligent robots for the on-orbit operation requirements of space stations, satellites, and large space structures.

\section{Space Intelligent Robot for Space Station Applications}

During the construction and operation of the ISS (Figure 1), a large-size space manipulator Canadarm II made by Canada was launched in 2001, which was mainly used to deploy, move, and capture payloads (Figure 2). It comprises seven joints, four cameras, two links, and two end-effectors with an arm span of $17.6 \mathrm{~m}$ and a total mass of $1640 \mathrm{~kg}$, capable of loading 116 tons of spacecraft. Four cameras can work continuously with $30 \mathrm{~Hz}$, and the rotation range of seven joints is $\pm 270^{\circ}$. It is symmetrical with respect to the elbow joint in the middle and has an end effector at each end of the robotic arm, which enables the robotic arm to move and crawl on the space station. Canadarm II can not only help the space station to dock the spacecraft but also use the manipulator to perform precise operational tasks in place of astronauts, which plays an important role on capturing large-mass cabins, docking assembly, and assisting astronauts out of the cabin [3]. However, its ability of dexterous manipulation is limited so that it still requires astronauts to perform dangerous extravehicular operations. Therefore, the on-orbit maintenance and operation of space station have an urgent demand for space intelligent robots.

In 2008, the Canadian Space Agency developed a special purpose manipulator system [4] (SPDM), also known as Dextre, which has a total mass of about $1560 \mathrm{~kg}$, a maximum load of $600 \mathrm{~kg}$, and a position accuracy of $2 \mathrm{~mm}$. The SPDM can rotate around the waist and has two 7-DOF robotic arms, with a total of $15 \mathrm{DOF}$. It does not have the ability to move and thus only can be installed at the end of Canadarm II. Canadarm II realizes a wide range of movement and rough positioning. The SPDM mainly completes precise operational tasks such as loading and unloading, handling, refueling, and operating small equipment to reduce the frequency of astronauts leaving the cabin. However, in the microgravity environment, the dual-arm robot SPDM is still difficult even to complete specific preset tasks [5], and some astronauts are still required to perform dangerous extravehicular operations.

After the successful verification of the spacecraft intersection and docking technology in the Tiangong No. 1 and No. 2 Space Laboratories, the core module of Chinese space station was successfully launched in April 2021. Chinese robotic manipulator system (Figure 3(b)) consists of a large space robotic arm and a small space robotic arm. The large space manipulator adopts the joint configuration scheme of "3DOF shoulder +1-DOF elbow +3-DOF wrist," a length of $10.2 \mathrm{~m}$, a total mass of $800 \mathrm{~kg}$, and a load of 25 tons of spacecraft. The small space manipulator also has $7 \mathrm{DOF}$, with a length of $5.0 \mathrm{~m}$, a total mass of $400 \mathrm{~kg}$, and a load of 3 tons. This arm has higher positioning accuracy and can realize dexterous manipulation. The end adapters with standard mechanical, electrical, and communication interfaces are designed at both ends of the large manipulator. By docking with the target adapter on the cabin surface, it can crawl and transfer on the

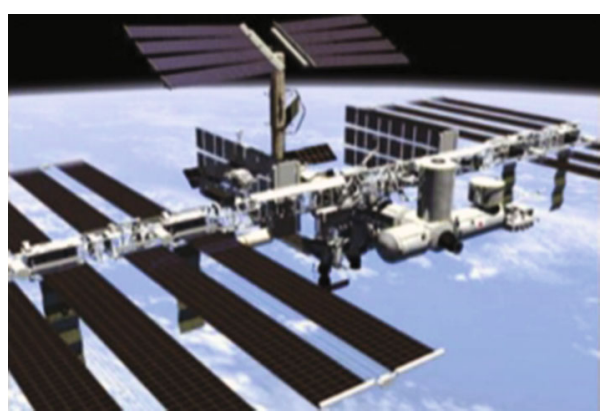

Figure 1: "Mir" International Space Station.

cabin surface, which can realize a wide range of cabin translocation, cargo handling, and extravehicular inspections and assist astronauts in exiting the cabin. Meanwhile, it can be combined with a small space manipulator to perform a series of space station maintenance and fine dexterous manipulation.

Since humanoid robots have the similar appearance to a human astronaut, there is no need to make any changes for adapting to space station, controlling robots like astronauts to perform human-like movements and operations, which is an effective way to assist or replace astronauts on duty in the space station. Thus, robot astronauts have been listed as one of the key development directions of space intelligent robots in major aerospace countries such as Europe, America, and Russia. In particular, the humanoid robot astronaut Robonaut2 (R2) developed by NASA has boarded the ISS in 2011 [6-8] (Figure 4(a)), where the technology of space intelligent operation is being verified. R2 is a highly integrated system of mechatronics, which has 42 independent DOF and more than 350 sensors, including two arms with 7 DOF, two hands with $12 \mathrm{DOF}$, a neck with $3 \mathrm{DOF}$, and a waist with $1 \mathrm{DOF}$. As the biggest feature, it has a flexible arm like a human, the maximum moving speed of the end effector is $2 \mathrm{~m} / \mathrm{s}$, and it can load $18 \mathrm{~kg}$ objects. Each dexterous hand has a gripping force of $2.3 \mathrm{~kg}$. R2 adopts an impedance-based distributed control method, which can complete more precise operation tasks. However, the motion mode and dynamic characteristics of robot astronauts in the microgravity environment are quite different from those in a ground gravity environment $[9,10]$. At the same time, the harsh environments including complex lighting conditions in space and high energy particle radiation have a great impact on sensors especially optical sensors [11], which results in great technical challenges for R2 dexterous manipulation at space stations [12].

The German Aerospace Center DLR has also developed a human-shaped mobile robot Justin [13] (Figure 4(b)). The diameter of its working space is $2.7 \mathrm{~m}$; it has a mass of about $200 \mathrm{~kg}$ and a rated load of $20 \mathrm{~kg}$. The robot has a total of 51 DOF. Its compliant, lightweight arm, and four-finger dexterous hand make it an ideal platform for studying dexterous manipulation. The motion detection sensor and stereo camera enable the robot to perform $3 \mathrm{D}$ reconstruction of the environment. Multiple driving DOF allow Justin to accomplish multiple tasks simultaneously in a multitask hierarchy. This robot is aimed at cooperating with humans to complete tasks, especially household chores and assisting astronauts in space work and other application fields. The Russian Aerospace Agency 


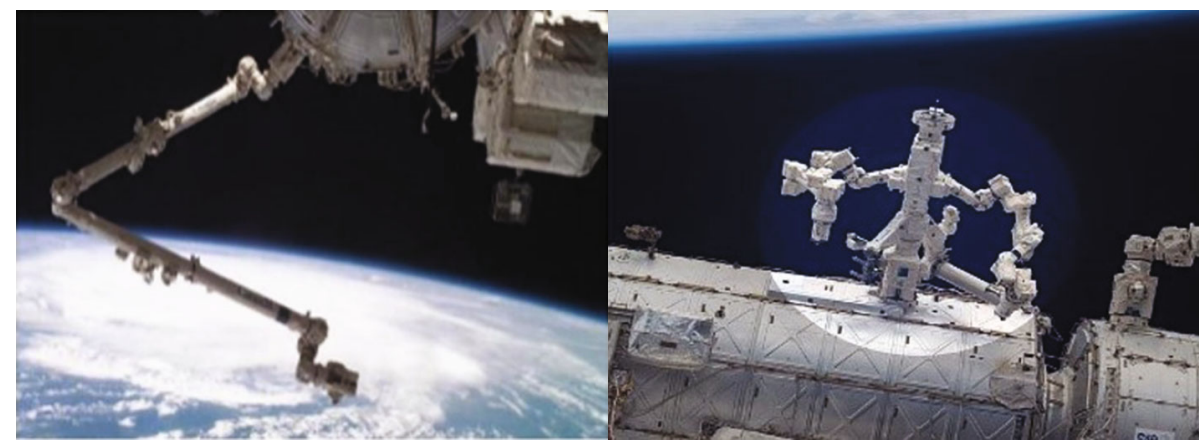

FIgURE 2: The Canadarm II and SPDM manipulator of ISS.

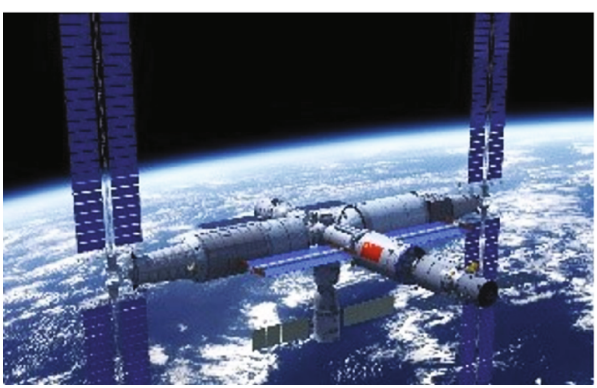

(a) China's space station

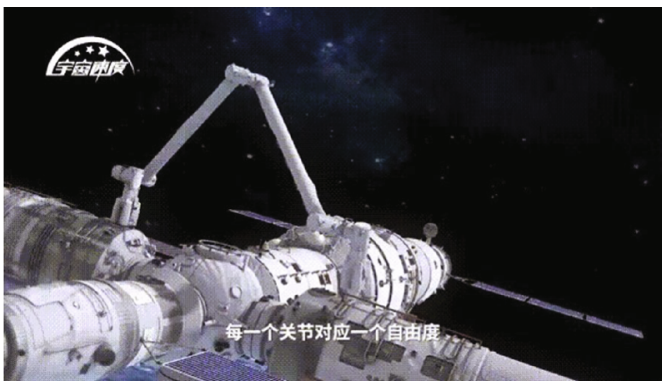

(b) The robotic manipulator system of CSS

Figure 3: China's space station and robotic manipulator system.

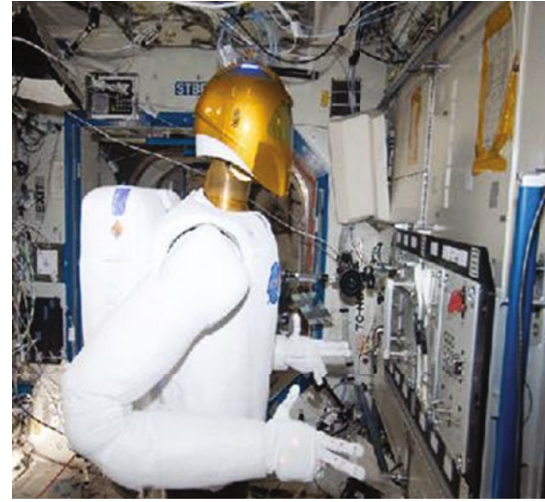

(a) Robonaut2

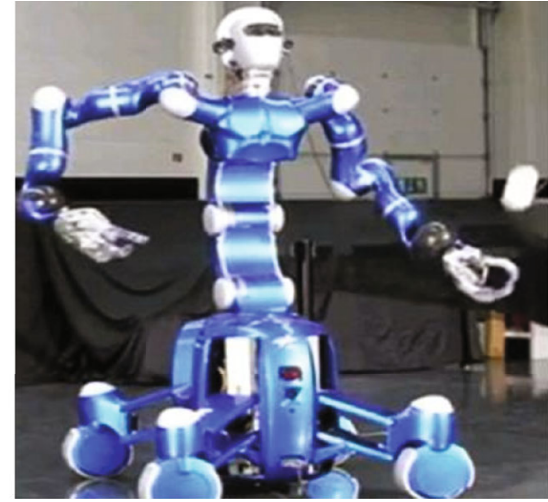

(b) Justin

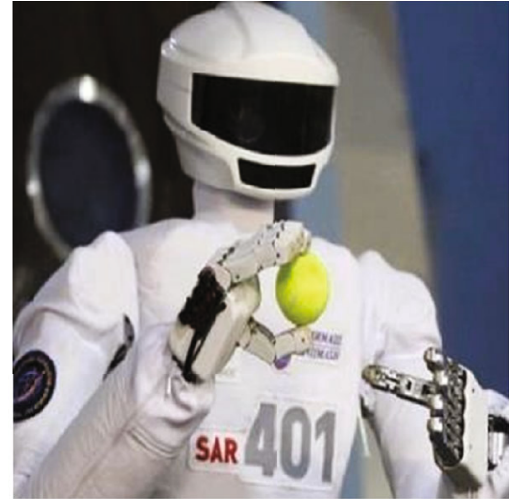

(c) SAR-401

FIgURE 4: Robot astronauts for on-orbit operation in the space station.

has developed SAR-400 and SAR-401 [14] (Figure 4(c)). SAR400 is a remote-controlled humanoid robot. One of the most obvious features is that it can share the sense of touch with people and achieve tactile feedback by using special gloves. Similarly, people can feed back their actions to the robot by wearing a head-mounted display and a sensory jacket. With these technologies, SAR-400 can achieve many complex and delicate operations in dangerous environments. Since the SAR-400 humanoid robot has no legs, it can be installed on the crane of the ISS to replace the astronauts to complete tasks such as tightening bolts, transferring cargo, inspecting the space station shell, and opening doors. SAR-401 is an improved model of SAR-400. SAR-401 has better performance of grasp- ing and more complex operations. It can complete more than 50 operations such as opening and closing the door and using electric drills. But until now, these two robot astronauts Justin and SAR-401 are still in the stage of ground research and verification.

The China Academy of Space Technology (CAST) and Shanghai Academy of Spaceflight Technology (SAST) have also developed two robot astronauts, respectively (Figures 5(a) and 5(b)). Both consist of a head, a torso, a neck with 3 DOF, a waist with $2 \mathrm{DOF}$, two arms with $7 \mathrm{DOF}$, and multi-DOF five-finger dexterous hands. The maximum load of the end of the arm is $10 \mathrm{~kg}$. The objective is to be used to on-orbit maintenance services and more space-exploration activities such as 


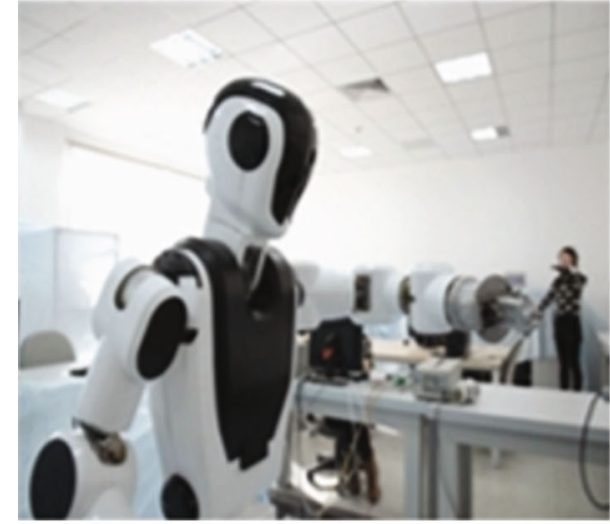

(a) The robot astronaut developed by CAST

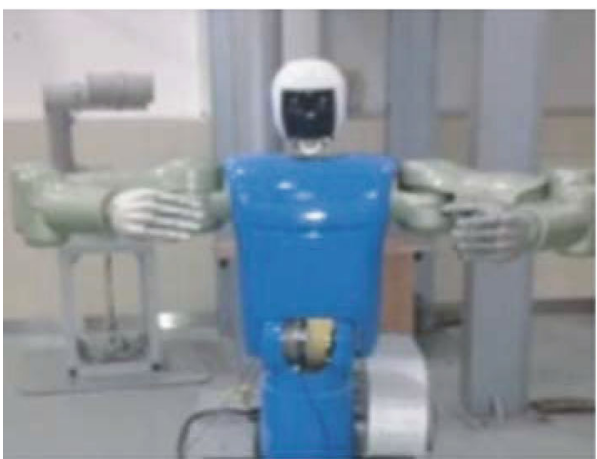

(c) The robot astronaut developed by HIT

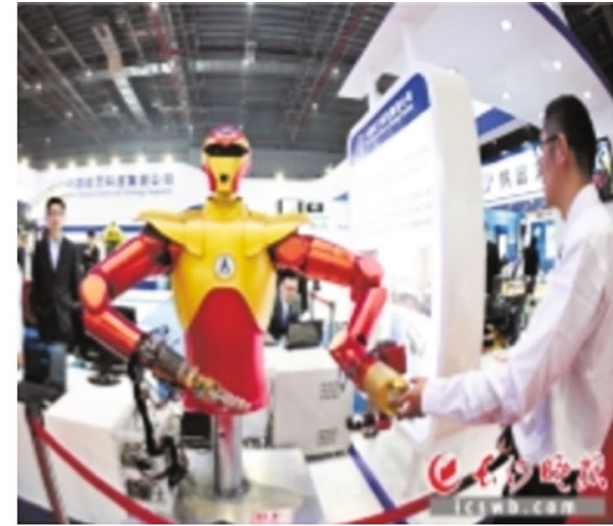

(b) The robot astronaut developed by SAST

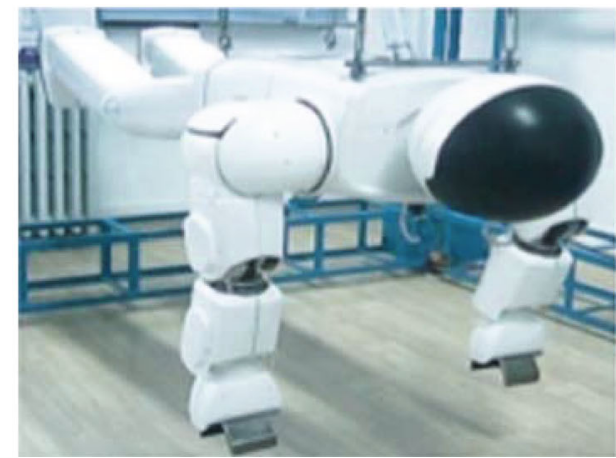

(d) The robot astronaut developed by BIT

FIgURE 5: Some robot astronauts developed by China.

space-station experiments and manned lunar missions [15]. The robot astronaut developed by Harbin Institute of Technology (HIT) (Figure 5(c)) also has two 7-DOF humanoid robotic arms and two multi-DOF five-finger dexterous hands with a maximum load of $10 \mathrm{~kg}$ at the end of the arm [16]. The arm was demonstrated and verified by the man-machine on-orbit maintenance operation in the Tiangong No. 2 Space Laboratory. The robot astronaut ASTROBOT developed by Beijing Institute of Technology (BIT) (Figure 5(d)) weighs about $50 \mathrm{~kg}$. It has $2 \mathrm{DOF}$ on the head and 6 DOF for each arm. The coordinated control algorithm of the master-slave arm enables the robot to achieve bionic climbing motion in the microgravity environment. Meanwhile, a control method of contact force-based feedforward compensation is adopted to ensure the stability of the climbing movement outside the cabin. In addition, a series of image enhancement algorithms and human-like dexterous manipulation algorithms have been proposed to realize the compliant installation and maintenance tasks of extravehicular components under space special lighting [17-19]. At present, Chinese robotic astronauts are still in the stage of breaking through key technologies and experimental verification on the ground.

The robot astronaut is a typical representative of intelligent robots for space station applications and is a research hotspot in space intelligent robots. However, it is difficult to carry out a wide range of stable movements and safe dex- terous manipulation in a small microgravity space [20], and it is also difficult to effectively verify the 3D motion of the robot astronaut on the ground [21,22]. At present, robot gait planning and dexterous manipulation based on human motion mechanism have made great progress [23-25]. However, there is a huge difference between the motion mechanism of humanoid robot in the microgravity environment and those on the ground. How to form a parameterized representation of the law and study the theory of motion planning and control adapted to space environment is a scientific problem that needs to be solved urgently about robot astronauts. Meanwhile, machine vision is an important basis for robotic manipulation, and the image filtering, recognition, and accurate positioning methods of the current machine vision have made great progress $[26,27]$. Unfortunately, there is a strong contrast between the shadow area and the illuminated area in the space, and the mirror-like coating of the space device may cause multiple refraction and reflection of light, high-energy particle radiation, and other complex interference environments, which seriously interfere with the features of the operating objects and challenge the existing image processing algorithms [28]. For example, the R2 robot astronaut of the ISS is equipped with 4 RGB cameras and an infrared TOF camera on the head to provide accurate depth information for improving the measurement accuracy [29]. Even so, it is difficult for R2 to overcome the interference of the complex space environment. In 2016, NASA 


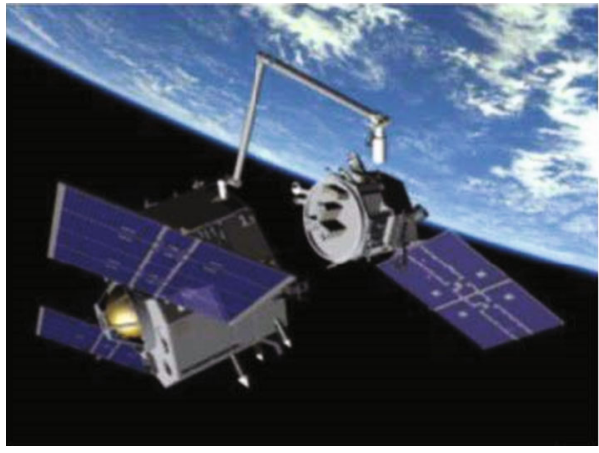

(a) Satellite capture

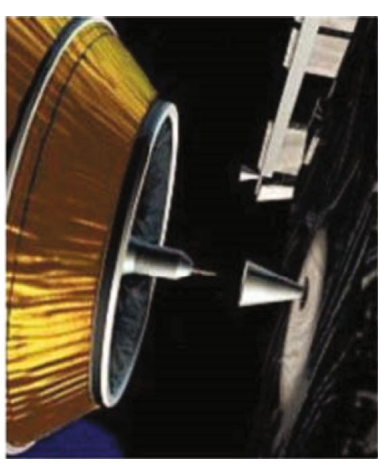

(b) Docking mechanism

Figure 6: The “Orbital Express” program of USA.

collected image-processing algorithms from all over the world to help R2 robustly recognize and accurately locate handles, tools, and other targets [11].

In summary, with the development of intelligent robot technology, the space-station intelligent robot has also been improved in terms of stable motion, dexterous manipulation, and precise sensing and measurement. In the future, it is very potential to study the theories and methods of human-like stable motion and dexterous manipulation by cross-integration of bionics and robotics, which can transfer human skills to robotic systems.

\section{Space Intelligent Robot for On-Orbit Maintenance of Satellites}

The main tasks of the intelligent robot for on-orbit maintenance of satellites are to repair faulty satellites (such as solar panels and antennas), replacement of components, and fuel supply. The main way is to approach the target satellite under the guidance of vision or radar and use the robotic arm to capture and maintain it, which provides an important guarantee for the safe operation of the satellite in orbit. "Orbital Express" (Figure 6) proposed by DARPA in 1999 is aimed at verifying the service capabilities of on-orbit satellites such as capture, maintenance and fuel replenishment, and the effectiveness of space operations. This plan consists of two satellites: one is the Autonomous Space Transport Robotic Orbiter (ASTRO) satellite produced by Boeing as the tracking satellite, and the other is the NEXT Generation Serviceable Satellite (NEXTSat) built by Ball Aerospace as the target satellite. ASTRO is equipped with an advanced video guidance sensor (AVGS), which uses the difference of two images formed under different wavelengths of light to eliminate background stray light and noise interference and then autonomously locate the NEXTSat satellite. Afterwards, NEXTSat is moved to the area of the capture envelope, and these two satellites are connected by a docking mechanism [30, 31]. During the maintenance phase, ASTRO can perform visual inspection on NEXTSat antennas, interface planes, solar panels, and other components and can replace components independently with the visual servobased robotic arm. The "Orbital Express" program successfully demonstrates some key technologies required for the autono-

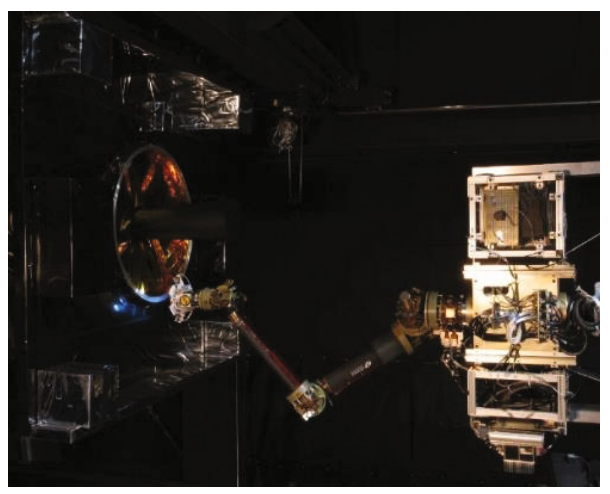

FIgURE 7: The FREND project of USA.

mous maintenance of satellites in orbit, including the technology of autonomous rendezvous, the technology of orbit control, capture and docking, the technology of on-orbit refueling, and the technology of on-orbit module replacement.

The "Orbital Express" program successfully achieved the capture and repair of a satellite equipped with a specific docking interface, but it was unable to operate any noncooperative targets. In 2002, the United States launched a project on "spacecraft for the universal modification of orbits" (SUMO), renamed as FREND (front-end robotics enabling near-term demonstration) in 2006. The main purpose of this project is to develop a space robot that docks with spacecraft in orbit and to verify the ability to automatically dock, capture, refuel, and repair noncooperative space targets without docking interface [32]. The focus of this task is to develop a 7-DOF space manipulator system (Figure 7), which can provide high position accuracy before the capture stage, with an end position accuracy of $\pm 2 \mathrm{~mm}$ and an angle accuracy of $\pm 0.4^{\circ}$. In the capture phase, it can perform compliant control and ensure the required rigidity when connecting with the spacecraft. At the same time, the robotic arm has a high degree of dexterity and can reach a working speed of $17 \mathrm{~cm} / \mathrm{s}(6 \mathrm{~cm} / \mathrm{s})$ along the axis of the end effector in $75 \%$ $(95 \%)$ of the working space. At present, it has been proved to be very reliable, and it is the new benchmark for spacelevel manipulators. 


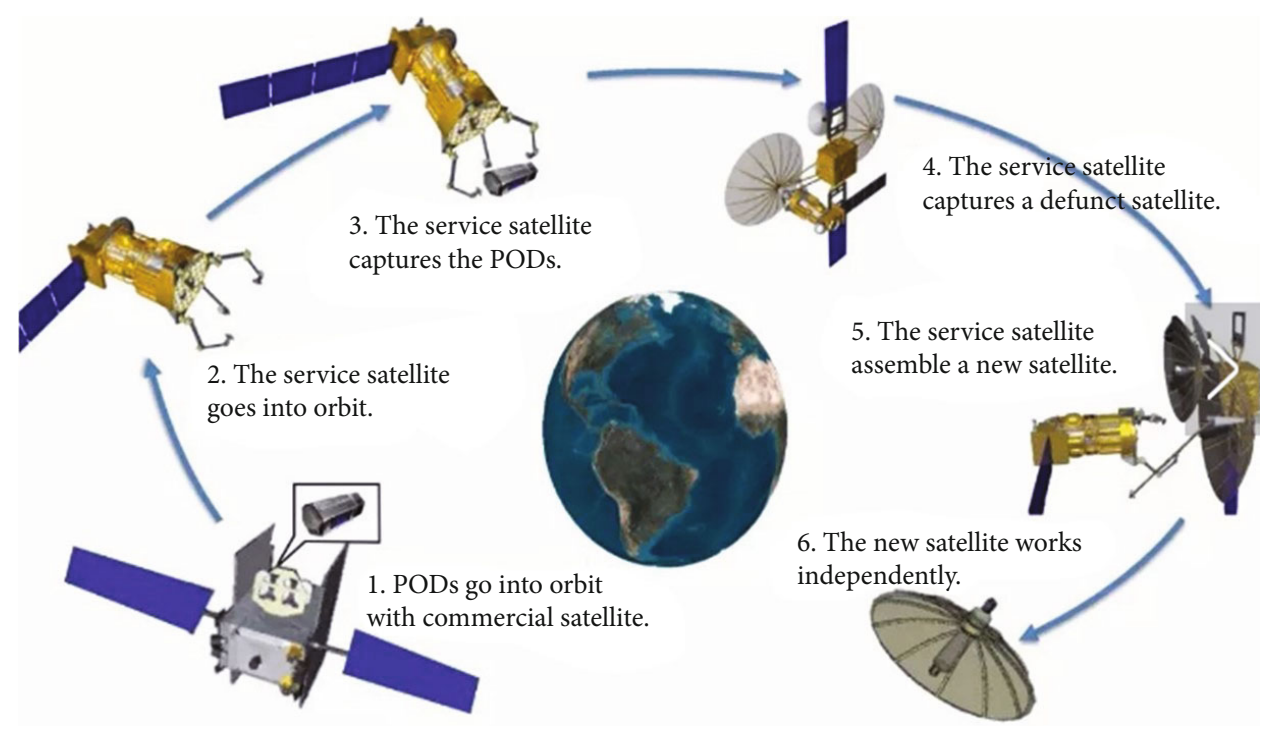

FIgURe 8: Execution process of Phoenix Spacecraft Servicing Program.

In 2012, DARPA launched the newest space technology research project "Phoenix Spacecraft Servicing Program" (PSSP), which is aimed at developing joint recovery technology to reuse functional components on satellites that are decommissioned or not working properly for recycling. Integrating these old components into the new space system significantly reduces the cost of new space equipment, which has huge commercial value. First, the Payload Orbital Delivery Systems (PODs) storing the satellite modular functional components (Satlets) are launched into space together with the satellite. When approaching the space intelligent robot, the PODs are ejected. The space robot approaches, grasps and stores PODs in the system devices. Then, the space intelligent robot moves to the abandoned satellite, intercepts the functional components (such as antenna) that can still work, and installs it on the modular functional components carried by the PODs. It is relatively cheap to build a "new" satellite in orbit [33]. The detailed procedures are shown in Figure 8. The key technologies involved in this project include the technology of PODs design, the technology of modular satellite design, the technology of robotic arm design, the technology of proximity measurement and identification, and the technology flexible control and teleoperation.

At the same time, Germany, Russia, Canada, and many other research institutions have also cooperated to do research on the free-flying robot system TECSAS, which is aimed at verifying the autonomous capture, docking, and on-orbit service technology of space robots to eliminate low-orbit satellites [34]. The project plans to launch a client satellite and a service satellite with a 7-DOF manipulator and a gripper system, as shown in Figure 9. The TECSAS project uses three control modes to capture free-floating and rolling satellites, namely autonomous mode, semiautonomous mode, and teleoperation mode. This project focuses on the technology of robotic arm capture, the technology of free-floating robot path planning, the technology of teleoperation singularity avoidance, and the technology of model-based parameter estima-

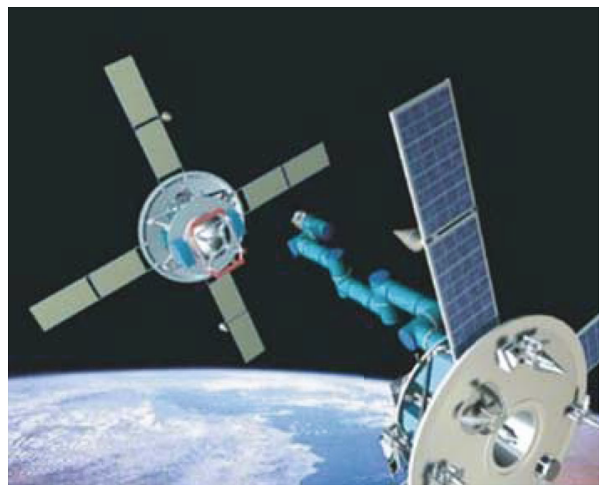

Figure 9: The free-flying robot system TECSAS of German.

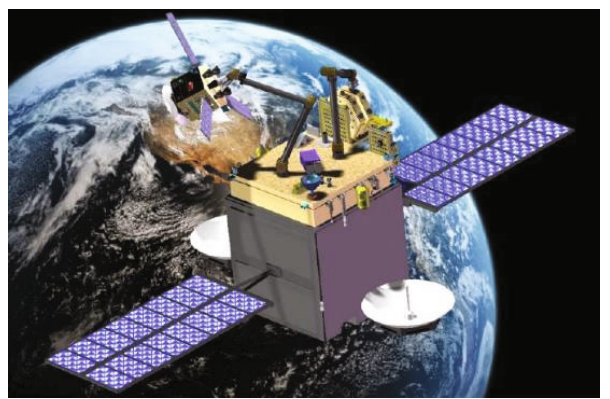

FIgURE 10: The dynamic simulation system of on-orbit satellite maintenance.

tion. However, the project only carried out on-orbit capture tests and was terminated due to changes of the plan.

The HIT, the China Academy of Launch Vehicle Technology (CALVT), and other institutions, respectively, have conducted various verification experiments on space robots to capture and repair satellites in orbit. They studied the design 
TABLE 1: Other related projects of space robots for on-orbit satellite maintenance.

\begin{tabular}{|c|c|c|c|c|}
\hline Project name & Country & Introduction of task & Time & Current status \\
\hline Ranger & USA & $\begin{array}{l}\text { Verify the technologies of ORU replacement, target } \\
\text { monitoring, and multiarm coordination }\end{array}$ & $1992-2001$ & Demonstration verification \\
\hline ETS-VII & Japan & $\begin{array}{l}\text { Verify the technologies of ORU replacement, } \\
\text { replenishment, and structural antenna assembly }\end{array}$ & 1992-1999 & On-orbit presentation \\
\hline Rotex & Germany & $\begin{array}{l}\text { Verify the technologies of multisensor manipulator and } \\
\text { compliant control }\end{array}$ & 1993 & On-orbit presentation \\
\hline XSS-10 & USA & $\begin{array}{l}\text { Verify the technologies of orbital maneuvering and } \\
\text { circumnavigation detection }\end{array}$ & 2003 & On-orbit presentation \\
\hline ROKVISS & Germany & $\begin{array}{l}\text { Verify the key technologies of lightweight robotic arms } \\
\text { in real space environments }\end{array}$ & 2004 & On-orbit presentation \\
\hline RRM & USA & $\begin{array}{l}\text { Verify the technologies of remote operation-based fuel } \\
\text { filling and fault maintenance }\end{array}$ & 2011-present & On-orbit presentation \\
\hline
\end{tabular}

modeling, parameter identification, visual servo-based position control, and compliance control of on-orbit maintenance system for space manipulators. The BIT has completed the scene simulation of satellite on-orbit maintenance operations, the dynamic simulation of the dual-arm robot, and the development and verification of a rapid replacement device adapted to the satellite on-orbit maintenance. The dynamic simulation of satellite capture, disassembly, and assembly process is shown in Figure 10.

The United States, Germany, Japan, Canada, and other countries have also conducted a large number of other related experiments (Table 1), which has promoted the rapid development and progress of space intelligent robot technology [35-45].

However, in the harsh space environment of high-speed flight and microgravity, it is a grand challenge for the design of high-precision rigid-flexible coupling manipulators, the technology of precise safe and dexterous manipulation, the technology of multirobot collaborative control, the technology of rapid tool change, and tooling system design. Meanwhile, in order to improve the efficiency of satellite onorbit assembly and maintenance, the modular satellites and cellular satellites are also a promising research direction. Overall, the space robots for satellite-oriented on-orbit assembly and maintenance system have great economic and social value, which is significant for the further promotion of human exploration and utilization of space resources.

\section{Space Intelligent Robot for On-Orbit Assembly of Space-Oriented Large- Scale Structures}

In the future, large-scale structures and facilities in space will greatly expand the horizons of mankind and will become an important way for mankind to explore and utilize space resource. However, due to its huge size and the difficulty of human on-orbit construction in the space, most of the large-scale structural facilities will be assembled and maintained on-orbit by space robots [46]. Modular assembly and on-orbit manufacturing are the main ways to gradually

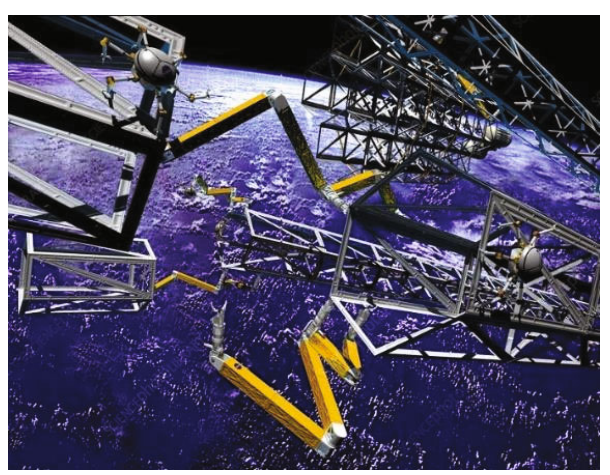

FIGURE 11: On-orbit assembly by Skyworker.

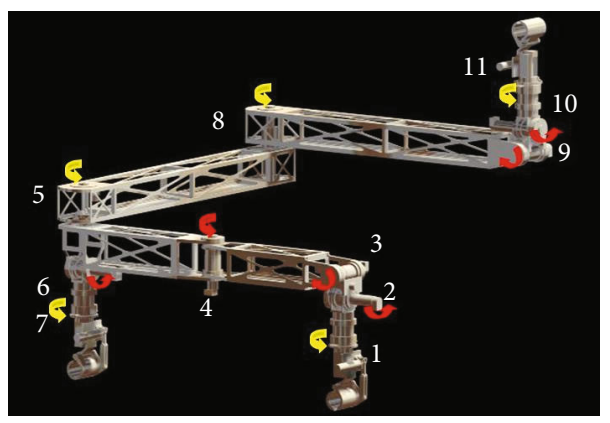

Figure 12: The joint configuration of Skyworker.

assemble and manufacture large-scale spatial structures from formed components or raw materials in the orbit [47], both of which are currently in the verification or planning stage. The Skyworker (Figure 11), a robot designed by Carnegie Mellon University, is an attached mobile robot that can move by applying a reaction force to objects without grabbing hooks or handrails. It has three functions: on-orbit assembly, detection, and maintenance. Skyworker mainly serves the transportation, assembly, and maintenance tasks of large payloads, such as on-orbit assembly of solar cell arrays [48].

Specifically, Skyworker has eleven joints, made up of four different types (Figure 12). All joints are connected with 


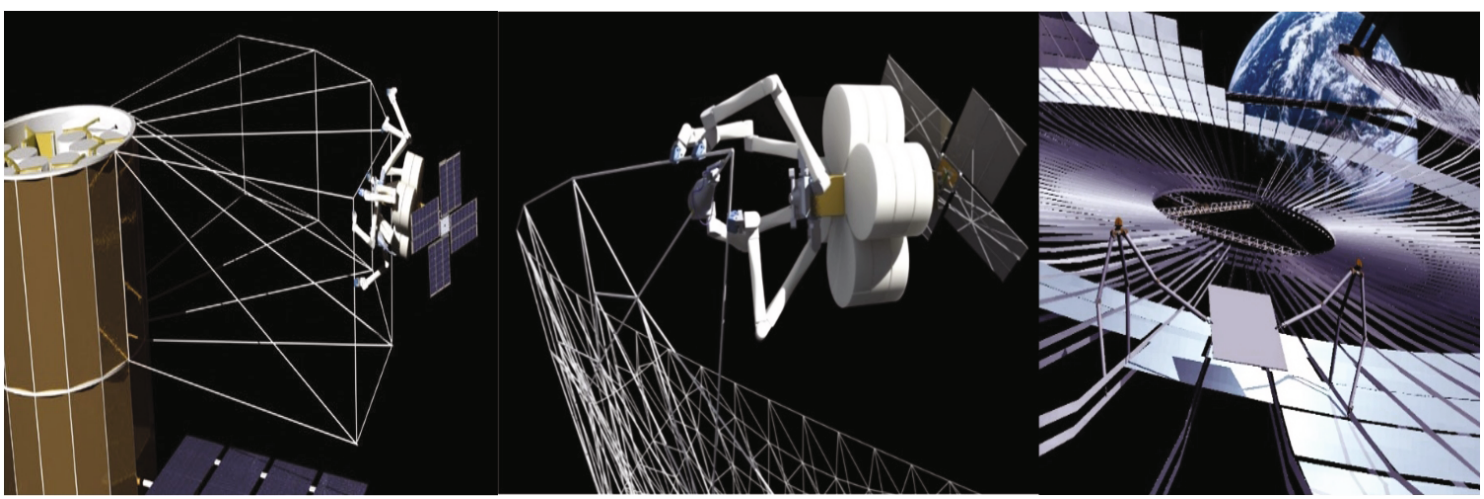

Figure 13: Manufacture of large-scale space structure by SpiderFab robot.

the same powertrain design, which minimizes independent components and achieves a modular configuration. Skyworker is designed to mainly perform two types of operations: manipulating and transporting payloads. When manipulation is required, the robot connects to the structure with an end gripper and manipulates with the other gripper. In this case, Skyworker is a 9-DOF robotic arm. When transportation is performed, the payload ranges from kilograms to tons, and its transportation distance reaches half a kilometer. The robot uses the PC104 stack and a distributed network of PIC-based motor controller for control. The controller runs the PID algorithm at $20 \mathrm{kHz}$, which can achieve high-precision motor position and speed control.

The current spacecraft construction mode that builds and integrates all components on the ground and launch them into orbit as a whole is actually expensive and time-consuming. Tethers Unlimited, Inc. (TUI) of America launched the SpiderFab project in 2012 and began to develop new spacemanufacturing technologies. This project mainly uses the $3 \mathrm{D}$ printer and materials carried by the multiarm space robot to print large trusses on orbit and assemble them on orbit to form a large-scale system [49], as shown in Figure 13. At present, TUI has built a truss-manufacturing robot on the ground, which can produce trusses at a speed of $5 \mathrm{~cm} / \mathrm{min}$. These trusses can be assembled into spacecraft structures and other systems in space. In the future, it may only need to launch raw materials and then build the large-scale system by the SpiderFab robot system in orbit [50], which will greatly save costs. Rob Hoyt, the chief scientist of TUI, said that SpiderFab robot can help humans build large radio antennas, spacecraft architectures, and solar arrays in the next ten years. The long-term goal of this technology is to ultimately achieve recycling of resources, to build infrastructure in space, and to support human exploration and utilization of space resources deeply.

For the on-orbit assembly and construction of ultralarge high-performance space antennas, NASA has proposed an on-orbit assembly plan based on the space shuttle, as shown in Figure 14. In this scheme, each unit is independent of each other, is unfolded one by one after being transported to the space, and is then docked. Alternatively, a few units can be spliced into groups on the ground, and after they

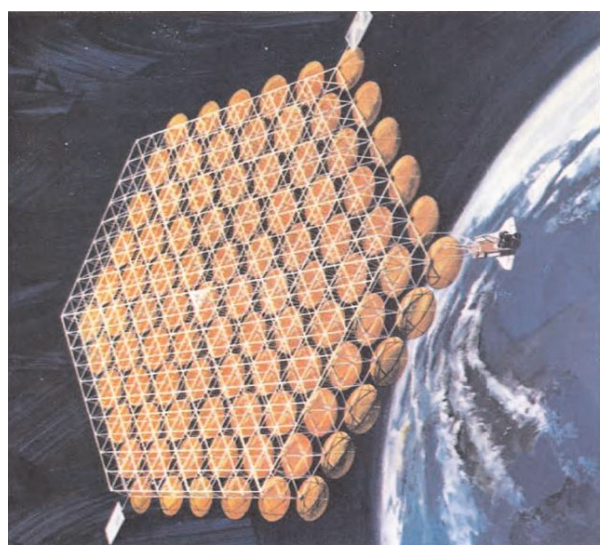

FIgURE 14: The on-orbit assembly and construction of ultralarge space antennas.

are launched into space, they finally are spliced to form a larger array [51].

The James Webb Space Telescope (JWST) [52] is a giant space telescope jointly developed by NASA, the European Space Agency, and the Canadian Aerospace Agency to enable a broad range of investigations across the fields of astronomy and cosmology. The diameter of the primary mirror is as high as 6.5 meters, and the area of the primary lens is 7.3 times that of Hubble Telescope [53]. Since the structure is very complicated and the diameter is larger than that of the launch rocket, the primary mirror is divided into 18 hexagonal segments (Figure 15). These segments are folded and launched into the space. Once they enter the predetermined orbit, they will complete deployment, assembly, and positioning correction in orbit under the control of high-precision micromotors and sensors to ensure that they can produce clear images [54]. But due to research funding and reliability requirements, the plan has been delayed beyond October 2021.

In the construction of space-based solar power stations (SSPS), the complex structure and huge volume also make it difficult to assemble them on the ground and launch them into orbit as a whole. Therefore, the technology of on-orbit assembly is very important for practical applications, which is also significant in the field of space exploration, commerce, and national defense. Scientists of China Academy of Space Technology have 


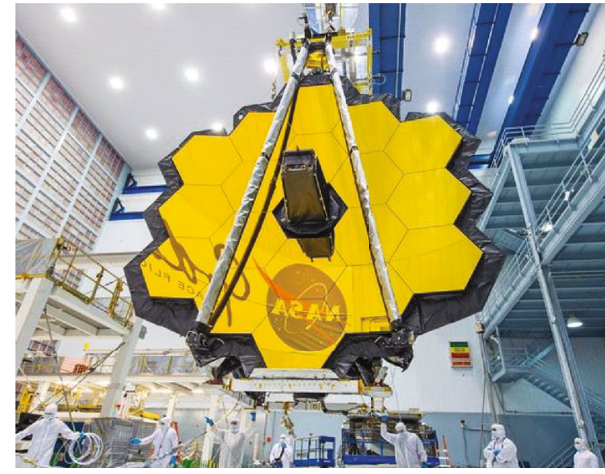

Figure 15: The James Webb Space Telescope.

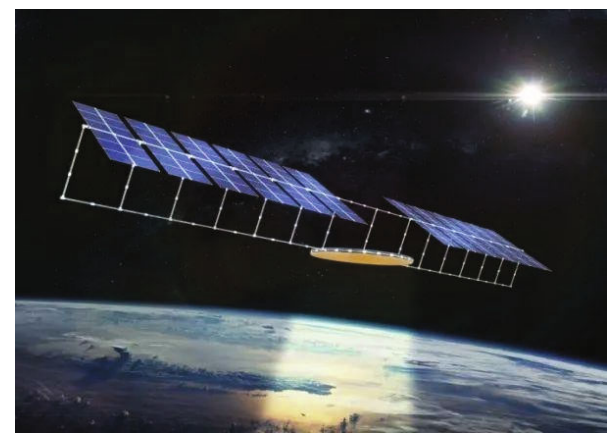

FIGURE 16: The concept design on multirotary joints SPS.

proposed the design scheme of two-stage symmetric reflected SSPS [55] and the concept design on multirotary joints SPS [56] (Figure 16). Xidian University has proposed an innovative concentrator system of the space solar power station SSPS-OMEGA [57]. The scheme optimizes the material of the concentrating system and the layout of reflectors and demonstrates the feasibility of on-orbit assembly. The research of space on-orbit assembly construction in China started late, and the current research is still mainly focused on the cabin docking and module replacement technology in the early stage. The manufacturing and assembly of large-scale space equipment are still in its infancy, but the on-orbit manufacturing and assembly is undoubtedly one of the important plans of China's aerospace in intelligent manufacturing. For example, China first validated the technology of $3 \mathrm{D}$ printing in space in May 2020, realizing 3D printing of continuous carbonfiber-reinforced composites in space [58].

Overall, space large-aperture antennas, large-aperture space telescopes, and large-scale solar power stations are of great significance to human beings for space exploration and space resource utilization. However, due to the limitation of the size of the carrier and the rocket carrying capacity, it is difficult to launch the large-scale structure directly into the space, and it is also difficult for astronauts to carry out on-orbit assembly of large-scale spacecraft due to physiological limitations. Therefore, on-orbit construction of high-mass, large-size, and highcomplexity spatial structures is the main development direction and research hotspot in the future. The core technologies include large inertia load handling system, large-range fast and stable moving mechanism, micronano high-precision space measurement system, and special tools and systematic equipment.

\section{Conclusion}

Space intelligent robot is an inevitable choice to improve the level of space automation technology. The major aerospace powers of the world have made many explorations in the development strategies, implementation plans, and key technologies of space intelligent robots and achieved a series of successes. It mainly includes the technologies of robotic manipulators and humanoid intelligent robots for space station applications, the robot technologies of on-orbit satellite maintenance robot, and the robot technologies of on-orbit assembly for large space structures. China is also conducting comprehensive demonstration and research on the applications and key technologies of space intelligent robots. However, in complex and harsh environments such as microgravity and strong radiation, the design of high-precision rigid-flexible coupling manipulators, highprecision dexterous and safe manipulation, multirobot collaborative control, and on-orbit assembly and maintenance of large equipment still face huge challenges. In future research, it is urgent to overcome these key scientific issues.

\section{Data Availability}

The data used to support the findings of this study are included within the article.

\section{Conflicts of Interest}

The authors declare that they have no conflicts of interest.

\section{Authors' Contributions}

X. Huang and Z. Jiang wrote the manuscript. Z. Jiang and $\mathrm{H}$. Li conceived the idea of this review and supervised the study. $\mathrm{X}$. Cao investigated the related study. M. Ceccarelli revised the manuscript. All authors discussed the results and contributed to the final version of the manuscript.

\section{Acknowledgments}

This work is supported in part by the National Key Research and Development Program of China under Grant no. 2018YFB1305300, the China Postdoctoral Science Foundation under Grant nos. 2020TQ0039 and 2021M700425, and the National Natural Science Foundation of China under Grant nos. 61733001, 62103054, U2013602, 61873039, U1913211, and U1713215.

\section{References}

[1] W. Bluethmann, R. Ambrose, M. Diftler et al., "Robonaut: a robot designed to work with humans in space," Autonomous Robots, vol. 14, no. 2/3, pp. 179-197, 2003.

[2] C. Tan, Q. Zhao, Y. Cao et al., "Investigation on effects of gradually increased Coriolis stimulus vestibular rehabilitation 
training," Space Medicine \& Medical Engineering, vol. 26, no. 1, pp. 20-23, 2013.

[3] W. Xu, J. Zhang, H. Qian, Y. Chen, and Y. Xu, "Identifying the singularity conditions of Canadarm2 based on elementary Jacobian transformation," in IEEE International Conference on Intelligent Robots and Systems, pp. 795-800, Tokyo, Japan, 2013.

[4] R. Mukherji, D. A. Ray, M. Stieber, and J. Lymer, "Special purpose dexterous manipulator (SPDM) advanced control features and development test results," in The 6th International Symposium on Artificial Intelligence, Robotics and Automation in Space, Quebec, Canada, 2001.

[5] O. Ma, J. Wang, S. Misra, and M. Liu, "On the validation of SPDM task verification facility," Journal of Robotic Systems, vol. 21, no. 5, pp. 219-235, 2004.

[6] M. A. Diftler, J. S. Mehling, M. E. Abdallah et al., "Robonaut 2-the first humanoid robot in space," in IEEE International Conference on Robotics and Automation, pp. 2178-2183, Shanghai, China, 2011.

[7] L. B. Bridgwater, C. A. Ihrke, M. A. Diftler et al., "The Robonaut 2 hand-designed to do work with tools," in IEEE International Conference on Robotics and Automation, pp. 3425-3430, Saint Paul, MN, USA, 2012.

[8] J. Wright, "Robonaut 2 Getting his Space Legs [EB/OL]," 2014, https://www.nasa.gov/mission_pages/station/main/robonaut .html.

[9] Y. Umetani and K. Yoshida, "Resolved motion rate control of space manipulators with generalized Jacobian matrix," IEEE Transactions on Robotics and Automation, vol. 5, no. 3, pp. 303-314, 1989.

[10] Y. Kazuya, "Engineering test satellite VII flight experiments for space robot dynamics and control: theories on laboratory test beds ten years ago, now in orbit," International Journal of Robotics Research, vol. 22, no. 5, pp. 321-335, 2003.

[11] R. Lewis, "Robonaut Vision Tool Manipulation Challenge," 2016, https://www.nasa.gov/feature/robonaut-vision-toolmanipulation-challenge.

[12] J. Badger, A. Hulse, R. Taylor, A. Curtis, D. Gooding, and A. Thackston, "Model-based robotic dynamic motion control for the Robonaut 2 humanoid robot," in 2013 13th IEEE-RAS International Conference on Humanoid Robots (Humanoids), pp. 62-67, Atlanta, GA, USA, 2013.

[13] C. Borst, T. Wimbock, F. Schmidt et al., "Rollin'Justin-mobile platform with variable base," in IEEE International Conference on Robotics and Automation, pp. 1597-1598, Kobe, Japan, 2009.

[14] "Russian experts have developed a SAR-401 simulation robot that is expected to work for astronauts [EB/OL]," 2015, http://www.chinanews.com/gj/2015/03-09/7112002.shtml.

[15] "Robot astronaut 'XiaoTian' debut [EB/OL]," 2015, http://zqb .cyol.com/html/2015-11/04/nw.D110000zgqnb_20151104_401.htm.

[16] J. Xia, Research on kinematic solution and avoiding collision for Robonaut arm, Harbin Institute of Technology, 2015.

[17] Z. H. Jiang, S. Liu, H. Li, Q. Dong, X. Chen, and Q. Huang, "Mechanism design and system control for humanoid space robot movement using a simple gravity-compensation system," International Journal of Advanced Robotic Systems, vol. 10, no. 11, p. 389, 2013.

[18] B. Wei, Z. H. Jiang, H. Li, Q. Dong, W. Ni, and Q. Huang, "Adaptive impedance controller for a robot astronaut to climb stably in a space station," International Journal of Advanced Robotic Systems, vol. 13, no. 3, p. 81, 2016.

[19] Q. Dong, Robot astronaut control method of climbing motion and compliance manipulation in space, Beijing Institute of Technology, 2015.

[20] Y. Mo, Stability control method and dynamics modeling of space manipulator, Beijing Institute of Technology, 2016.

[21] A. Flores-Abad, O. Ma, K. Pham, and S. Ulrich, "A review of space robotics technologies for on-orbit servicing," Progress in Aerospace Sciences, vol. 68, pp. 1-26, 2014.

[22] O. Ma, M. Zebenay, and T. Boge, "Control of industrial robots for hardware-in-the-loop simulation of satellite docking," in SPIE Defense, Security, and Sensing, vol. 8044, International Society for Optics and Photonics, 2011.

[23] C. G. Valdivia, A. B. Ortega, E. Q. M'rquez, and M. O. Salazar, "Development of a biped robot based on dynamic walking: design, simulation and construction," in International Conference on Intelligent Environments, pp. 95-102, Guanajuato, Mexico, 2012.

[24] A. M. Dolla and R. D. How, "The highly adaptive SDM hand: design and performance evaluation," The International Journal of Robotics Research, vol. 29, no. 5, pp. 585-597, 2010.

[25] Y. Ogura, K. Shimomura, H. Kondo et al., "Human-like walking with knee stretched, heel-contact and toe-off motion by a humanoid robot," in IEEE International Conference on Intelligent Robots and Systems, pp. 3976-3981, Beijing, China, 2006.

[26] S. Y. Chen, "Kalman filter for robot vision: a survey," IEEE Transactions on Industrial Electronics, vol. 59, no. 11, pp. 4409-4420, 2012.

[27] S. Lowry, N. Sunderhauf, P. Newman et al., "Visual place recognition: a survey," IEEE Transactions on Robotics, vol. 32, no. 1, pp. 1-19, 2016.

[28] L. B. Cheng, Z. H. Jiang, H. Li, B. Wei, and Q. Huang, "Targettools recognition method based on an image feature library for space station cabin service robots," Robotica, vol. 34, no. 4, pp. 925-941, 2016.

[29] G. V. Tzvetkova, "Robonaut 2: mission, technologies, perspectives," Journal of Theoretical and Applied Mechanics, vol. 44, no. 1, pp. 97-102, 2014.

[30] D. A. Whelan, E. A. Adler, S. B. Wilson III, and G. M. Roesler Jr., "DARPA orbital express program: effecting a revolution in space-based systems," in Small Payloads in Space, San Diego, CA, United States, 2000.

[31] M. Wright, "Orbital express space operations architecture program," in Proceedings of SPIE-The International Society for Optical Engineering, vol. 5088, pp. 1-9, Orlando, Florida, United States, 2003.

[32] T. Debus and S. Dougherty, "Overview and performance of the front-end robotics enabling near-term demonstration (FREND) robotic arm," in AIAA Infotech@Aerospace Conference, Seattle, Washington, 2009.

[33] L. Gunn, J. Palmer, J. Leung et al., "DARPA Phoenix Payload Orbital Delivery System: progress towards small satellite access to GEO," in Proceedings of Small Satellite Conference, North Logan, Utah, United States, 2015.

[34] B. Sommer, "Unmanned on-orbit servicing in the German Space Program - the TECSAS Mission," in International Astronautical Congress of the International Astronautical Federation, Bremen, Germany, 2013.

[35] G. Gefke, C. R. Carignan, and B. E. Roberts, "Ranger telerobotic shuttle experiment: a status report," in Proceedings of SPIE- 
The International Society for Optical Engineering, pp. 123-132, Boston, MA, United States, 2002.

[36] K. Yoshida, "Engineering Test Satellite VII Flight experiments for space robot dynamics and control: theories on laboratory test beds ten years ago, now in orbit," in Experimental Robotics VII, Springer-Verlag, 2003.

[37] T. M. Davis and D. Melanson, "XSS-10 microsatellite flight demonstration program results," in Proceedings of SPIE-The International Society for Optical Engineering, vol. 5419, Orlando, Florida, United States, 2004.

[38] J. Chen and F. Chen, "Robots perform space refueling show," Space Exploration, vol. 3, pp. 30-33, 2013.

[39] C. Kaiser, F. Sjöberg, J. M. Delcura, and B. Eilertsen, "SMARTOLEV-An orbital life extension vehicle for servicing commercial spacecrafts in GEO," Acta Astronautica, vol. 63, no. 1-4, pp. 400-410, 2008.

[40] G. Hirzinger, B. Brunner, J. Dietrich, and J. Heindl, "ROTEXthe first remotely controlled robot in space," in Proceedings of the 1994 IEEE International Conference on Robotics and Automation, San Diego, CA, USA, 2002.

[41] C. Preusche, D. Reintsema, K. Landzettel, and G. Hirzinger, "Robotics component verification on ISS ROKVISSpreliminary results for telepresence," in IEEE/RSJ International Conference on Intelligent Robots \& Systems, Beijing, China, 2005.

[42] G. Visentin and D. L. Brown, "Robotics for geostationary satellite servicing," Robotics and Autonomous Systems, vol. 23, no. 1-2, pp. 45-51, 1998.

[43] R. Boumans and C. Heemskerk, "The European Robotic Arm for the International Space Station," Robotics and Autonomous Systems, vol. 23, no. 1-2, pp. 17-27, 1998.

[44] M. Nagatomo, T. Mitome, K. Kawasaki, N. Ezawa, and K. Kasuga, "MFD Robot Arm and Its Flight Experiment," in Sixth ASCE Specialty Conference and Exposition on Engineering, Construction, and Operations in Space, Albuquerque, New Mexico, United States, 1998.

[45] N. Sato and Y. Wakabayashi, "JEMRMS design features and topics from testing," in Proceedings of the IEEE International Conference on Artificial Intelligence, Robotics and Automation in Space, pp. 1298-1307, Quebec, Canada, 2001.

[46] Y. Xu, B. Brown, S. Aoki, and T. Kanade, "Mobility and manipulation of a light-weight space robot," Robotics and Autonomous Systems, vol. 13, no. 1, pp. 1-2, 1994.

[47] N. G. Cui, P. Wang, J. F. Guo, and X. Cheng, "A review of onorbit servicing," Journal of Astronautics, vol. 28, no. 4, pp. 805811, 2007.

[48] P. J. Staritz, S. Skaff, C. Urmson, and W. Whittaker, "Skyworker: a robot for assembly, inspection and maintenance of large scale orbital facilities," in IEEE International Conference on Robotics \& Automation, Seoul, Korea (South), 2001.

[49] R. P. Hoyt, "SpiderFab: an architecture for self-fabricating space systems," in Aiaa Space Conference \& Exposition, San Diego, CA, 2013.

[50] Y. H. Feng, "Spider robots promote on-orbit manufacturing in space," Defense Point, vol. 6, pp. 60-61, 2015.

[51] X. F. Ma, Y. Li, and Y. Xiao, "Development and tendency of large space deployable antenna reflector," Space Electronic Technology, vol. 15, no. 2, pp. 16-26, 2018.

[52] R. Garner, "About the James Webb Space Telescope [EB/OL]," 2018, https://jwst.nasa.gov/about.html.
[53] “James Webb Space Telescope [EB/OL]," 2021, https://en .wikipedia.org/wiki/James_Webb_Space_Telescope.

[54] W. Wilkie, R. Williams, G. Agnes, and B. Wilcox, "Structural feasibility analysis of a robotically assembled very large aperture optical space telescope," Structural Dynamics and Materials Conference, Collection of Technical Papers-AIAA/ASME/ ASCE/AHS/ASC Structures, vol. 2, Honolulu, Hawaii, 2007.

[55] X. L. Meng, X. Chen, X. L. Xia, and G. L. Dai, "Energy transfer characteristics of symmetrical space two-stage reflected solar concentrator," Journal of Astronautics, vol. 34, no. 9, pp. 1288-1294, 2013.

[56] X. B. Hou, L. Wang, X. H. Zhang, and L. Zhou, "Concept design on multi-rotary joints SPS," Journal of Astronautics, vol. 36, no. 11, pp. 1332-1338, 2015.

[57] Y. A. Yang, D. U. Bao-Yan, H. U. Jin, L. I. Xun, Z. H. Yi-Qun, and F. A. Jian-Yu, "SSPS-OMEGA: a new concentrator system for SSPS," Chinese Space Science and Technology, vol. 5, pp. 18-23, 2014.

[58] X. S. Quan, “China First Verified 3D Printing Technology in Space [EB/OL]," 2020, http://news.sciencenet.cn/htmlnews/ 2020/5/439521.shtm. 